\title{
The optimal order for the p-th moment of sums of independent random variables with respect to symmetric norms and related combinatorial estimates ${ }^{\ddagger}$
}

\author{
Marius Junge \\ University of Illinois at Urbana-Champaign \\ 1409 West Green Street \\ Urbana, Illinois 61801,USA \\ e-mail: junge@math.uiuc.edu
}

\begin{abstract}
For $n$ independent random variables $f_{1}, . ., f_{n}$ and a symmetric norm \|\|$_{X}$ on $\mathbb{R}^{n}$, we show that for $1 \leq p<\infty$

$$
\begin{aligned}
& \frac{1}{2+4 \sqrt{2}}\left(\left(n \int_{0}^{\frac{1}{n}} h^{*}(s)^{p} d s\right)^{\frac{1}{p}}+\left\|\sum_{i=1}^{n}\left(n \int_{\frac{i-1}{n}}^{\frac{i}{n}} h^{*}(s) d s\right) e_{i}\right\|_{X}\right) \\
& \leq\left(\int\left\|\sum_{i=1}^{n} f_{i} e_{i}\right\|_{X}^{p} d \mu\right)^{\frac{1}{p}} \\
& \leq \frac{c_{0} p}{1+\ln p}\left(\left(n \int_{0}^{\frac{1}{n}} h^{*}(s)^{p} d s\right)^{\frac{1}{p}}+\left\|\sum_{i=1}^{n}\left(n \int_{\frac{i-1}{n}}^{\frac{i}{n}} h^{*}(s) d s\right) e_{i}\right\|_{X}\right) .
\end{aligned}
$$

Here

$$
h(t, \omega)=\sum_{i=1}^{n} 1_{\left[\frac{i-1}{n}, \frac{i}{n}\right)}(t) f_{i}(\omega)
$$

is the disjoint sum of the $f_{i}$ 's and $h^{*}$ is the non-increasing rearrangement. Similar results (where $L_{p}$ is replaced by a more general rearrangement invariant function space) were obtained first by Litvak, Gordon, Schütt and Werner for Orlicz spaces $X$ and independently by S. Montgomery-Smith for general $X$ but without an explicit analysis of the order of growth for the constant in the upper estimate. The order $\frac{p}{1+\ln p}$ is optimal and obtained from combinatorial estimates for doubly stochastic matrices. The result extends to Lorentz-norms $l_{f, q}$ on $\mathbb{R}^{n}$ under mild assumptions on $f$. We give applications to the theory of noncommutative $L_{p}$ spaces.
\end{abstract}

\footnotetext{
${ }^{\ddagger}$ Keywords: Symmetric norms, independent random variables, combinatorial probability; 2000 Mathematics Subject Classification: 46B09, 60G50, 60C05, 47L20
} 


\section{Introduction and Notation}

The interaction between Banach space theory and probabilistic methods have a long tradition and Rosenthal's inequality [Ro], extended by Burkholder [Bu] to general martingales, is an example for an inequality motivated by Banach space theory with a significant impact in probability. Since then there has been a big progress in calculating the expectation of the norm of independent variables in particular by Johnson, Schechtman, Zinn and Johnson, Schechtman [JS], Kwapien, Szulga [KSz], Hitczenko [Hi], Hitczenko/MontgomerySmith [HMS] and many others. Motivated by embedding problems for non-commutative $L_{p}$ spaces, we calculate the $p$-norm of the sum of independent random variables with respect to symmetric norms ${ }^{1}$. This extends recent results of Gordon, Litvak, Schütt and Werner. Very recently, similar results have been obtained by Montgomery-Smith [MS] (but by different techniques and without an analysis of the order of constant involved. The starting point of our approach an article of Geiss [GE] using certain $K$-functional from interpolation theory.

More precisely, we calculate the $p$-th moment of the sum of $n$ independent random variables $f_{1}, . ., f_{n}$ with respect to a symmetric norm \|\|$_{X}$ on $\mathbb{R}^{n}$, i.e. a norm satisfying

$$
\left\|\sum_{i=1}^{n} \varepsilon_{i} x_{\pi(i)} e_{i}\right\|_{X}=\left\|\sum_{i=1}^{n} x_{i} e_{i}\right\|_{X}
$$

for all coefficients $\alpha_{i}$, changes of signs $\varepsilon_{i} \pm 1$ and permutations $\pi:\{1, . ., n\} \rightarrow\{1, . ., n\}$. Our results follow a general philosophy: Independent variables behave like disjoint variables. Although our results hold for all such symmetric norms, it turns out that only few classical norms are really relevant for this investigation. Indeed, certain $K$-functionals $\mid \_k$ between the $\ell_{1}$ and the $\ell_{\infty}$ norm (see section 1 ) and the weak- $\ell_{1}$ norm $\ell_{1, \infty}$ (see section 2 and section 3 ).

Let us recall the notation of the non-increasing rearrangement. Given $n$ independent random variables $f_{1}, . ., f_{n}$, we consider the disjoint sum

$$
h(t, \omega)=\sum_{i=1}^{n} 1_{\left[\frac{i-1}{n}, \frac{i}{n}\right)}(t) f_{i}(\omega) .
$$

Then non-increasing rearrangement $h^{*}$ is defined by

$$
h^{*}(s)=\inf \{t \mid \operatorname{Prob}(|h|>t) \leq s\} .
$$

A starting point of our approach is the following theorem of S. Geiss [GE, proof of theorem 3.4] based on previous work of Johnson and Schechtman [JS].

Theorem 0.1 (S. Geiss) Let $f_{1}, . ., f_{n}$ be independent ranodm variables, then

$$
2^{-\frac{1}{p}}\left(n \int_{0}^{\frac{1}{n}} h^{*}(s)^{p} d s\right)^{\frac{1}{p}} \leq\left(\int_{\Omega} \sup _{i}\left|f_{i}\right|^{p} d \mu\right)^{\frac{1}{p}} \leq 2^{1-\frac{1}{p}}\left(n \int_{0}^{\frac{1}{n}} h^{*}(s)^{p} d s\right)^{\frac{1}{p}} .
$$

\footnotetext{
${ }^{1}$ The first version of this article dates back to 1998
} 
On the other hand, we are motivated by some combinatorial estimates of Kwapien and Schütt [KSI, KSII]. In contrast to our situation they consider

$$
\int_{\pi \text { Permutation }}\left\|\sum_{i=1}^{n} \alpha_{i \pi(i)}\right\|_{X} d \pi
$$

for the $\ell_{p}$-norms or more generally Orlicz spaces and arbitrary matrices $\alpha_{i j}$. This is also the starting point of [GLSW]. In general results for the permutation group or independent coefficients are very similar. We refer to [MSe] for further information on averages with respect to the group of permutations. Our main result is the following.

Theorem 0.2 Let $1 \leq p<\infty$ and \|\|$_{X}$ be a 1-symmetric, 1-unconditional norm on $\mathbb{R}^{n}$ with normalized unit vectors $e_{i}$. Then for all independent random variables $f_{1}, \ldots, f_{n}$ on a probability space $(\Omega, \mu)$

$$
\begin{aligned}
& \frac{1}{2+4 \sqrt{2}}\left(\left(n \int_{0}^{\frac{1}{n}} h^{*}(s)^{p} d s\right)^{\frac{1}{p}}+\left\|\sum_{i=1}^{n}\left(n \int_{\frac{i-1}{n}}^{\frac{i}{n}} h^{*}(s) d s\right) e_{i}\right\|_{X}\right) \leq\left(\int_{\Omega}\left\|\sum_{i=1}^{n} f_{i} e_{i}\right\|_{X}^{p} d \mu\right)^{\frac{1}{p}} \\
& \leq \frac{c_{0} p}{1+\ln p}\left(\left(n \int_{0}^{\frac{1}{n}} h^{*}(s)^{p} d s\right)^{\frac{1}{p}}+\left\|\sum_{i=1}^{n}\left(n \int_{\frac{i-1}{n}}^{\frac{i}{n}} h^{*}(s) d s\right) e_{i}\right\|_{X} .\right.
\end{aligned}
$$

The order of the constant $\frac{p}{1+\ln p}$ is optimal.

For the lower estimate, it suffices to consider the supremums norm and the sequence of ||$_{k}$-norms (see section 1). In this paper we give a selfcontained proof of the second inequality (called upper bound) only using Rosenthal's inequality and new combinatorial tools. It turns out that an upper bound the norm in Lorentz space $\ell_{1, \infty}$ implies the general case (see section 2 for this.) Indeed, the key estimate is of combinatorial nature and we believe it is of independent interest.

Theorem 0.3 There exists a constant $c_{0}$ with the following property. Let $\left(\mu_{i j}\right)_{i j}$ be a doubly stochastic matrix, i.e.for all $i, j$

$$
\sum_{k} \mu_{i k}=1=\sum_{k} \mu_{k j} .
$$

Then

$$
\left(\sum_{j_{1}, . ., j_{n}=1}^{n}\left(\sup _{r} \frac{1}{r} \operatorname{card}\left\{i \mid j_{i} \leq r\right\}\right)^{p} \prod_{k=1}^{n} \mu_{k j_{k}}\right)^{\frac{1}{p}} \leq c_{0} \frac{p}{1+\ln p} .
$$

The order of growth is optimal.

As an application of our result, we obtain a result for the Schatten class $S_{X}$ associated to a symmetric sequence space

$$
S_{X}=\left\{a \in B\left(\ell_{2}\right) \mid\|a\|_{S_{X}}=\left\|\sum_{k} s_{k}(a) e_{k}\right\|_{X}<\infty\right\} .
$$

Here $s_{k}(a)=\lambda_{k}\left(\sqrt{a^{*} a}\right)$ are the singular values of $a$. 
Theorem 0.4 Let $1 \leq p<\infty$ and $X$ be a symmetric sequence space. Then there is a von Neumann algebra $N$ such that $X$ embeds into some $L_{p}(N)$ iff there is a (possibly different) von Neumann algebra $N$ such that $S_{X}$ embeds into $L_{p}(N)$.

We will use standard notation from probability. $|A|$ denotes the cardinality of a set. Moreover, $c_{0}$ is used (as above) for an absolute constant varying in each occurrence. We use $a \sim_{c} b$ if $\frac{1}{\sqrt{c}} \leq \frac{a}{b} \leq \sqrt{c}$. The space of sequences converging to 0 is denoted by $c_{o}$. The paper is organized as follows. We prove the lower estimate in section 1. In the second part, we show how the upper estimate can be deduced from Rosenthal's inequality and the combinatorial estimate. Section 4 is devoted to the elementary proof of the combinatorial estimate. Theorem 0.4 and further applications are contained in section 5.

Acknowledgment: I want to thank Stefan Geiss for helpful discussions leading to the conjecture of theorem 0.2 and Y. Gordon for bringing to his attention the articles [GLSW] and $[\mathrm{MS}]$.

\section{The lower estimate}

In this section, we will prove the lower estimate in Theorem 0.2. The arguments are simialr as in [GLSW] and [MS], but we give a proof with a 'concrete' estimate. We assume that \|\|$_{X}$ is a 1-unconditional, 1-symmetric and normalized norm. In our investigation, the following norms are of particular interest

$$
\begin{aligned}
\|x\|_{p} & =\left(\sum_{i=1}^{n}\left|x_{i}\right|^{p}\right)^{\frac{1}{p}}, \\
\|x\|_{p, \infty} & =\sup _{k} k^{\frac{1}{p}} x_{k}^{*}, \\
|x|_{k} & =\sum_{j=1}^{k} x_{j}^{*} .
\end{aligned}
$$

Here $\left(x_{j}^{*}\right)_{j=1}^{n}$ given by

$$
x_{j}^{*}:=\inf \left\{t||\left\{i|| x_{i} \mid>t\right\} \mid<j\right\}
$$

is the non-increasing rearrangement of $x$. Indeed, ||$_{k}$ is equivalent to the $K$-functional between $\ell_{1}$ and $\ell_{\infty}$ at the value $k$. We will need the following lemma of [KSI] which is the analogue of Geiss' theorem 0.1.

Theorem 1.1 (Kwapien Schütt) Let $\left(\alpha_{i j}\right)_{i j=1, . ., n}$ be an $n \times n$ matrix, $\alpha_{1}^{*}, . ., \alpha_{n}^{*}$ be its non-decreasing rearrangement and $d \pi$ the normalized counting measure on the group $\Pi_{n}$ of permutations of $\{1, . ., n\}$, then

$$
\frac{1}{2} \frac{1}{n} \sum_{j=1}^{n} \alpha_{j}^{*} \leq \int_{\Pi_{n}} \sup _{i=1, . ., n}\left|\alpha_{\pi(i) i}\right| d \pi \leq \frac{1}{n} \sum_{j=1}^{n} \alpha_{j}^{*} .
$$


Corollary 1.2 Let $1 \leq k \leq n$ and $x=\left(x_{1}, . ., x_{n}\right) \in \mathbb{R}^{n}$, then

$$
\frac{1}{4} \frac{1}{k} \sum_{j=1}^{k} x_{j}^{*} \leq \int_{\Pi_{n}} \sup _{i \leq \frac{n}{k}}\left|x_{\pi(i)}\right| d \pi \leq 2 \frac{1}{k} \sum_{j=1}^{k} x_{j}^{*} .
$$

Proof: Apply theorem 1.1 to $\alpha_{i j}=\left\{\begin{array}{ll}x_{i} & \text { if } j \leq \frac{n}{k} \\ 0 & \text { else }\end{array}\right.$. Let $m=\left[\frac{n}{k}\right] \geq 1$ be the smallest integer with $k m \leq n \leq k(m+1)$, then the rearrangement $\alpha^{*}$ satisfies

$$
\frac{1}{n} \sum_{j=1}^{n} \alpha_{j}^{*}=\frac{1}{n}\left(\left[\frac{n}{k}\right]\left(\sum_{i=1}^{k} x_{i}^{*}\right)+\left(n-k\left[\frac{n}{k}\right]\right) x_{k+1}^{*}\right) .
$$

By the monotonicity of the $x_{j}^{*}$, we get

$$
\begin{aligned}
\frac{1}{2} \frac{1}{k} \sum_{i=1}^{k} x_{i}^{*} & \leq\left[\frac{n}{k}\right] \frac{1}{n} \sum_{i=1}^{k} x_{i}^{*} \leq \frac{1}{n}\left(\left[\frac{n}{k}\right]\left(\sum_{i=1}^{k} x_{i}^{*}\right)+\left(n-k\left[\frac{n}{k}\right]\right) x_{k+1}^{*}\right) \\
& \leq \frac{1}{k} \sum_{i=1}^{k} x_{i}^{*}+\frac{1}{n}\left(k\left(\left[\frac{n}{k}\right]+1\right)-k\left[\frac{n}{k}\right]\right) \frac{1}{k} \sum_{i=1}^{k} x_{i}^{*} \\
& \leq\left(1+\frac{k}{n}\right) \frac{1}{k} \sum_{i=1}^{k} x_{i}^{*} .
\end{aligned}
$$

Proof of the lower estimate in Theorem 0.2: As usual, the trick for the lower estimate is an appropriate Abel summation. Indeed, let $x_{1} \geq x_{2} \geq x_{3} \geq \cdots \geq x_{n} \geq 0$ be a non-increasing sequence. It is well-known and easy to check, that

$$
\|x\|_{X}=\sup _{y \in C} \sum_{i=1}^{n} x_{i} y_{i}
$$

where $C$ consists of those vectors in the unit ball $B_{X^{*}}$ which are again positive and non-increasing. In this case, we have

$$
\sum_{i=1}^{n} x_{i} y_{i}=y_{n} \sum_{i=1}^{n} x_{i}+\sum_{k=1}^{n-1}\left(y_{j}-y_{j+1}\right) \sum_{i=1}^{k} x_{i} .
$$

In other terms for an arbitrary vector $x$

$$
\|x\|_{X}=\sup _{y \in C} y_{n} \sum_{i=1}^{n} x_{i}^{*}+\sum_{k=1}^{n-1}\left(y_{k}-y_{k+1}\right) \sum_{i=1}^{k} x_{i}^{*} .
$$

Now, we consider independent random variables $f_{1}, . ., f_{n}$ and the random vector

$$
x(\omega)=\left(f_{1}(\omega), \cdots, f_{n}(\omega)\right) .
$$


Using (1), we obtain

$$
\begin{aligned}
& \int_{\Omega}\left\|\sum_{i=1}^{n} f_{i}(\omega) e_{i}\right\|_{X} d \mu \\
& \quad=\int_{\Omega} \sup _{y \in C}\left(y_{n} \sum_{i=1}^{n} x_{i}^{*}(\omega)+\sum_{k=1}^{n-1}\left(y_{k}-y_{k+1}\right) \sum_{i=1}^{k} x_{i}^{*}(\omega)\right) d \mu(\omega) \\
& \quad \geq \sup _{y \in C} y_{n} \int_{\Omega}\left(\sum_{i=1}^{n} x_{i}^{*}(\omega)\right) d \mu(\omega)+\sum_{k=1}^{n-1}\left(y_{k}-y_{k+1}\right) \int_{\Omega}\left(\sum_{i=1}^{k} x_{i}^{*}(\omega)\right) d \mu(\omega) \\
& \quad=\sup _{y \in C} y_{n} \int_{\Omega}\left\|\sum_{i=1}^{n} f_{i} e_{i}\right\|_{1} d \mu+\sum_{k=1}^{n-1}\left(y_{k}-y_{k+1}\right) \int_{\Omega}\left|\sum_{i=1}^{k} f_{i} e_{i}\right|_{k} d \mu .
\end{aligned}
$$

This reduces the problem to the investigation of norms ||$_{k}$. Let us define the increasing sequence

$$
s_{j}=\left(n \int_{\frac{j-1}{n}}^{\frac{j}{n}} h^{*}(s) d s\right) .
$$

According to Corollary 1.2, by independence and Fubini's theorem, we deduce from the proof of Geiss's inequality [GE, Theorem 3.4] and monotonicity of $h^{*}$

$$
\begin{aligned}
\frac{2}{k} \int_{\Omega}\left|\sum_{i=1}^{k} f_{i} e_{i}\right|_{k} d \mu & \geq \int_{\Omega} \int_{\Pi_{n}} \sup _{i \leq \frac{n}{k}}\left|f_{\pi(i)}(\omega)\right| d \pi d \mu=\int_{\Pi_{n}} \int_{\Omega} \sup _{i \leq \frac{n}{k}}\left|f_{\pi(i)}(\omega)\right| d \mu d \pi \\
& =\int_{\Omega} \sup _{i \leq \frac{n}{k}}\left|f_{i}(\omega)\right| d \mu \geq \frac{1}{\sqrt{2}}\left[\frac{n}{k}\right] \int_{0}^{\frac{1}{\left[\frac{n}{k}\right]}} h^{*}(s) d s \\
& \geq \frac{1}{2 \sqrt{2}} \frac{n}{k} \int_{0}^{\frac{k}{n}} h^{*}(s) d s=\frac{1}{2 \sqrt{2}} \frac{1}{k} \sum_{j=1}^{k}\left(n \int_{\frac{j-1}{n}}^{\frac{j}{n}} h^{*}(s) d s\right) \\
& =\frac{1}{2 \sqrt{2}} \frac{1}{k} \sum_{j=1}^{k} s_{j} .
\end{aligned}
$$

Combining this with the previous estimate, we obtain again from (1)

$$
\begin{aligned}
\int_{\Omega}\left\|\sum_{i=1}^{n} f_{i}(\omega) e_{i}\right\|_{X} d \mu & \geq \frac{1}{4 \sqrt{2}} \sup _{y \in C} y_{n}\left(\sum_{j=1}^{n} s_{j}\right)+\sum_{k=1}^{n-1}\left(y_{k}-y_{k+1}\right) \sum_{i=1}^{k} s_{i} \\
& =\frac{1}{4 \sqrt{2}}\left\|\sum_{i=1}^{n} s_{i} e_{i}\right\|_{X} .
\end{aligned}
$$

Trivially, we have $\|x\|_{X} \geq\|x\|_{\infty}$ and therefore Geiss' inequality [GE] concludes the proof

$$
\left(\int_{\Omega}\left\|\sum_{i} f_{i} e_{i}\right\|_{X}^{p} d \mu\right)^{\frac{1}{p}} \geq\left(\int_{\Omega}\left\|\sum_{i} f_{i} e_{i}\right\|_{\infty}^{p} d \mu\right)^{\frac{1}{p}} \geq 2^{-\frac{1}{p}}\left(n \int_{0}^{\frac{1}{n}} h^{*}(s)^{p} d s\right)^{\frac{1}{p}} .
$$




\section{The upper bound}

In this section, we will prove the upper bound. Given $n$ independent random variables $f_{1}, . ., f_{n}$, we may use the fact that for every monotone increasing function $g$

$$
\int g(f) d \mu=g(0)+\int_{0}^{\infty} g^{\prime}(s) \mu(|f|>s) d s=\int_{0}^{1} g\left(f^{*}(t)\right) d t .
$$

Therefore, we can assume that $f_{1}, . ., f_{n}$ are defined on $[0,1]^{n}$ and non-increasing. First, we split all the $f_{i}$ 's into three parts. Let $b=h^{*}\left(\frac{1}{n}\right)$ and

$$
f_{i}^{1}=f_{i} 1_{\left\{\left|f_{i}\right|>b\right\}} \quad, \quad f_{i}^{2}=\left(f_{i}-f_{i}^{1}\right) 1_{\left[0, \frac{1}{n}\right)} .
$$

We put $f_{i}^{3}=f_{i}-f_{i}^{1}-f_{i}^{2}$. The estimate for the first two parts uses Rosenthal's [Ro] inequality $(k \in\{1,2\})$

$$
\begin{aligned}
\left(\int\left\|\sum_{i=1}^{n} f_{i}^{k} e_{i}\right\|_{X}^{p} d \mu\right)^{\frac{1}{p}} & \leq\left(\int\left(\sum_{i=1}^{n}\left|f_{i}^{k}\right|\right)^{p} d \mu\right)^{\frac{1}{p}} \\
& \leq c(p)\left(\int \sum_{i=1}^{n}\left|f_{i}^{k}\right| d \mu+\left(\int \sup _{i}\left|f_{i}^{k}\right|^{p} d \mu\right)^{\frac{1}{p}}\right) .
\end{aligned}
$$

We note that according to [JSZ], we have $c(p) \leq \frac{c_{0} p}{1+\ln p}$. Let us observe that the two sets

$$
\begin{aligned}
& A^{1}=\left\{\left(t, s_{1}, \ldots, s_{n}\right) \mid f_{i}\left(s_{i}\right)>b\right\}=\left\{\left(t, s_{1}, \ldots, s_{n}\right)|| h\left(t, s_{1}, . ., s_{n}\right) \mid>h^{*}\left(\frac{1}{n}\right)\right\} \\
& A^{2}=\left\{\left(t, s_{1}, \ldots, s_{n}\right) \mid \frac{i-1}{n} \leq t<\frac{i}{n} \Rightarrow s_{i} \leq \frac{1}{n}\right\}
\end{aligned}
$$

have measure less than $\frac{1}{n}$ and therefore

$$
\frac{1}{n} \sum_{i=1}^{n}\left\|f_{i}^{k}\right\|_{1}=\int_{A^{k}}|h| \leq n^{\frac{1}{p}-1}\left(\int_{0}^{\frac{1}{n}} h^{*}(s)^{p} d s\right)^{\frac{1}{p}}=\frac{1}{n}\left(n \int_{0}^{\frac{1}{n}} h^{*}(s)^{p} d s\right)^{\frac{1}{p}} .
$$

Moreover, according to [GE, Theorem 3.4]

$$
\left(\int \sup _{i}\left|f_{i}^{k}\right|^{p} d \mu\right)^{\frac{1}{p}} \leq\left(\int \sup _{i}\left|f_{i}\right|^{p} d \mu\right)^{\frac{1}{p}} \leq 2^{1-\frac{1}{p}}\left(n \int_{0}^{\frac{1}{n}} h^{*}(s)^{p} d s\right)^{\frac{1}{p}} .
$$

Hence, by Hölders inequality for $k \in\{1,2\}$

$$
\left(\int\left\|\sum_{i=1}^{n} f_{i}^{k} e_{i}\right\|_{X}^{p} d \mu\right)^{\frac{1}{p}} \leq 4 c(p)\left(n \int_{0}^{\frac{1}{n}} h^{*}(s)^{p} d s\right)^{\frac{1}{p}} .
$$

The estimate of the third part uses the following proposition. 
Proposition 2.1 Let $X=\left(\mathbb{R}^{n},\|\|_{X}\right)$ be a symmetric sequence space, $\left(\alpha_{i j}\right)_{i, j=1, . ., n}$ be a matrix and $\alpha_{1}^{*}, \ldots, \alpha_{n^{2}}^{*}$ be the non-increasing rearrangement of the matrix, then

$$
\left(n^{-n} \sum_{j_{1}, \ldots, j_{n}=1}^{n}\left\|\sum_{k=1}^{n} \alpha_{k j_{k}} e_{k}\right\|_{X}^{p}\right)^{\frac{1}{p}} \leq c_{0} p\left\|\sum_{k=1}^{n} \alpha_{(k-1) n+1}^{*} e_{k}\right\|_{X}
$$

To conclude the proof of the upper estimate in Theorem 0.2 , we apply the Proposition to the matrix

$$
\alpha_{i j}=\sup _{\frac{j}{n}<s \leq \frac{j+1}{n}} f_{i}^{3}(s) .
$$

$\left(\alpha_{i n}=0.\right)$ For fixed $\omega=\left(s_{1}, . ., s_{n}\right)$ with $\frac{j_{i}}{n}<s_{i} \leq \frac{j_{i}+1}{n}$, we get

$$
\left\|\sum_{i=1}^{n} f_{i}^{3}\left(s_{i}\right) e_{i}\right\|_{X} \leq\left\|\sum_{i=1}^{n} \alpha_{i j_{i}} e_{i}\right\|_{X}
$$

The probability of the set where $s_{i} \in\left(\frac{j_{i}}{n}, \frac{j_{i}+1}{n}\right]$ is $\frac{1}{n}$ and for $s_{i} \leq \frac{1}{n}$ the variable $f_{i}^{3}\left(s_{i}\right)$ vanishes. Hence, we get

$$
\left(\int\left\|\sum_{i=1}^{n} f_{i}^{3} e_{i}\right\|_{X}^{p}\right)^{\frac{1}{p}} \leq\left(n^{-n} \sum_{j_{1}, ., j_{n}=1}^{n}\left\|\sum_{i=1}^{n} \alpha_{i j_{i}} e_{i}\right\|_{X}^{p}\right)^{\frac{1}{p}} \leq \frac{c_{0} p}{1+\ln p}\left\|\sum_{k=1}^{n} \alpha_{(k-1) n+1}^{*} e_{k}\right\|_{X} .
$$

On the other hand, let us consider the new variables

$$
\tilde{f}_{i}=\sum_{j=1}^{n} \alpha_{i j} 1_{\left[\frac{j-1}{n}, \frac{j}{n}\right)} .
$$

By definition $\tilde{f}_{i} \leq f_{i}$ and therefore

$$
\tilde{h}\left(t, s_{1}, . ., s_{n}\right):=\sum_{i=1}^{n} 1_{\left[\frac{i-1}{n}, \frac{i}{n}\right]} \tilde{f}_{i} \leq h .
$$

We observe that the non-increasing rearrangement of $\tilde{h}$ is the same as the non-increasing rearrangement of the matrix $\alpha$. For simplicity, let us assume that the values $\alpha_{i j}$ 's are all different from each other. These values appear in $\tilde{h}$ on the disjoint sets

$$
A_{i j}=\left\{\left(t, s_{1}, . ., s_{n}\right) \mid \frac{i-1}{n} \leq t<\frac{i}{n}, \frac{j-1}{n} \leq s_{i}<\frac{j}{n}\right\}
$$

of measure $\frac{1}{n^{2}}$. This implies for all $j=1, . ., n$

$$
\alpha_{j n+1}^{*}=\tilde{h}^{*}\left(\frac{j}{n}\right) .
$$

Together with

$$
\tilde{h}^{*}\left(\frac{j}{n}\right) \leq n \int_{\frac{j-1}{n}}^{\frac{j}{n}} \tilde{h}^{*}(s) d s \leq n \int_{\frac{j-1}{n}}^{\frac{j}{n}} h^{*}(s) d s
$$


we deduce from $\alpha_{1}^{*} \leq b=h^{*}\left(\frac{1}{n}\right)$ that

$$
\begin{aligned}
\left\|\sum_{k=1}^{n} \alpha_{(k-1) n+1}^{*} e_{k}\right\|_{X} & \leq \alpha_{1}^{*}+\left\|\sum_{k=2}^{n} h^{*}\left(\frac{k-1}{n}\right) e_{k}\right\|_{X} \\
& \leq h^{*}\left(\frac{1}{n}\right)+\left\|\sum_{k=2}^{n}\left(n \int_{\frac{k-2}{n}}^{\frac{k-1}{n}} h^{*}(s) d s\right) e_{k}\right\|_{X} \\
& \leq n \int_{0}^{\frac{1}{n}} h^{*}(s) d s+\left\|\sum_{k=1}^{n}\left(n \int_{\frac{k-1}{n}}^{\frac{k}{n}} h^{*}(s) d s\right) e_{k}\right\|_{X} \\
& \leq\left(n \int_{0}^{\frac{1}{n}} h^{*}(s)^{p} d s\right)^{\frac{1}{p}}+\left\|\sum_{k=1}^{n}\left(n \int_{\frac{k-1}{n}}^{\frac{k}{n}} h^{*}(s) d s\right) e_{k}\right\|_{X}
\end{aligned}
$$

This concludes the proof of (Proposition $2.1 \Rightarrow$ upper estimate in Theorem 0.2 ). The proof of Proposition 2.1 relies on the combinatorial estimate 0.3 and the following elementary observation.

Lemma 2.2 Let $x=\left(x_{1}, . ., x_{n}\right)$ be a positive non increasing sequence $y \in \mathbb{R}^{n}$ built by repetitions of the coordinates in $x$, i.e. for $y_{j} \in\left\{x_{1}, . ., x_{n}\right\}$ for all $1 \leq j \leq n$. If

$$
\beta_{i}:=\operatorname{card}\left\{j \mid y_{j}=x_{i}\right\}
$$

then

$$
\|y\| \leq 2 \max \left\{1, \sup _{r} \frac{1}{r} \sum_{i=1}^{r} \beta_{i}\right\}\|x\| .
$$

Proof: We assume

$$
\sum_{i=1}^{r} \beta_{i} \leq t r
$$

for all $r$ and $t \geq 1$. Let $1 \leq k \leq n$. In order to calculate $y_{k}^{*}$, we choose $r$ such that

$$
\sum_{i=1}^{r-1} \beta_{i}<k \leq \sum_{i=1}^{r} \beta_{i} .
$$

By the assumption on $y$, this means $y_{k}^{*}=x_{r}$. By assumption on $t$, we have

$$
k \leq \sum_{i=0}^{r} \beta_{i} \leq t r .
$$

In other terms $y_{k}^{*} \leq x_{r} \leq x_{\left[\frac{k}{t}\right]}$. Here we use the convention $[r]=1$ for $r<1$. In order to estimate the norm of $T_{t}(x)=x_{\frac{k}{t}}$, we fix a nonincreasing sequence $z$ in the unit ball of $X^{*}$. We use Abel summation for $\tilde{x}=T_{t}(x)$

$$
\langle\tilde{x}, z\rangle=z_{n}\left(\sum_{i=1}^{n} \tilde{x}_{i}\right)+\sum_{k=1}^{n-1}\left(z_{k}-z_{k+1}\right) \sum_{j=1}^{k} \tilde{x}_{j} .
$$


We fix $1 \leq k \leq n$, then

$$
\sum_{j=1}^{k} x_{\left[\frac{j}{t}\right]} \leq t x_{1}+\sum_{1 \leq i \leq \frac{k}{t}} t x_{i} \leq 2 t \sum_{i \leq k} x_{i}
$$

Hence, we deduce from $z_{k}-z_{k+1} \geq 0$ that

$$
\langle\tilde{x}, z\rangle \leq 2 t\left(z_{n}\left(\sum_{i=1}^{n} x_{i}\right)+\sum_{k=1}^{n-1}\left(z_{k}-z_{k+1}\right) \sum_{j=1}^{k} x_{j}\right) \leq 2 t\langle x, z\rangle \leq 2 t\|z\|_{X^{*}}\|x\|_{X} .
$$

This shows

$$
\|y\|_{X} \leq\left\|T_{t}(x)\right\|=\sup _{z}\left\langle T_{t}(x), z\right\rangle \leq 2 t\|x\| .
$$

Proof of Proposition 2.1: The combinatorial estimate will be shown in the next paragraph. We will show how it can be used to prove the Proposition. Using (1), we can again assume $\alpha_{i 1} \geq \alpha_{i 2} \geq \cdots \geq \alpha_{i n}$ for all $i=1, . ., n$. Moreover, by perturbation, we can assume that all the $\alpha_{i j}$ 's are different. For fixed $i, k$ we denote by $I_{i k}$ the interval in $[1, n]$ satisfying

$$
j \in I_{i k} \quad \Leftrightarrow \quad \alpha_{k n+1}^{*}<\alpha_{i j} \leq \alpha_{(k-1) n+1}^{*}
$$

We define

$$
\mu_{i k}:=\frac{1}{n} \operatorname{card}\left(I_{i k}\right) .
$$

Using the convention $\alpha+n^{2}+1=0$, we have

$$
\sum_{k=1}^{n} \mu_{i k}=1
$$

On the other hand, there are $n$ different values between $\alpha_{k n+1}^{*}$ and $\alpha_{(k-1) n+1}^{*}$, hence

$$
\sum_{i=1}^{n} \mu_{i k}=1
$$

Thus $\mu$ is a doubly stochastic matrix. For $j \in I_{i k}$, we can replace $\alpha_{i j}$ by the bigger value $\alpha_{(k-1) n+1}^{*}$. Let us denote this modified matrix by $\tilde{\alpha}$. Let us observe that the random variable

$$
\tilde{f}_{i}=\sum_{f=1}^{n} \tilde{\alpha}_{i j} 1_{\{j\}}
$$

on the probability space $\{1, . ., n\}$ with $\lambda(\{j\})=\frac{1}{n}$ satisfies

$$
\lambda\left(\tilde{f}_{i}=\alpha_{(k-1) n+1)}^{*}\right)=\mu_{i k} .
$$

Hence $f_{i}$ has the same distribution (and hence the same non-increasing rearrangement) as

$$
g_{i}=\sum_{k=1}^{n} \alpha_{(k-1) n+1}^{*} 1_{\{k\}} \quad \text { with respect to the measure } \mu_{i}(\{k\})=\mu_{i k} .
$$


Therefore, the assertion follows from an estimate of

$$
\left(\int_{\{1, . ., n\}^{n}}\left\|\sum_{i=1}^{n} g_{i}\left(j_{i}\right) e_{i}\right\|_{X}^{p} d \mu_{1}\left(j_{1}\right) \cdots d \mu_{n}\left(j_{n}\right)\right)^{\frac{1}{p}}
$$

For an individual element $\omega=\left(j_{1}, . ., j_{n}\right)$, we observe that $\left(g_{1}\left(j_{1}\right), \cdots, g_{n}\left(j_{n}\right)\right)$ only takes the values in the set $\alpha_{(k-1) n+1}^{*}$ for $k=1, . ., n$. Moreover, let $\beta_{k}$ be the cardinality of this occurrence, then

$$
\beta_{k}=\sum_{i=1}^{n} \delta_{k j_{i}}
$$

Hence, we get

$$
\frac{1}{r} \sum_{k=1}^{r} \beta_{k}=\frac{1}{r} \sum_{i=1}^{n} 1_{[1, r]}\left(j_{i}\right) .
$$

According to Lemma 2.2, we have

$$
\left\|\sum_{i=1}^{n} g_{i}\left(j_{i}\right) e_{i}\right\|_{X} \leq 2 \max \left\{1, \sup _{r} \frac{1}{r} \sum_{i=1}^{n} 1_{[1, r]}\left(j_{i}\right)\right\}\left\|\sum_{k=1}^{n} \alpha_{(k-1) n+1}^{*} e_{k}\right\|_{X} .
$$

Theorem 0.3 yields the assertion.

Remark 2.3 The typical example for the theorem is the (quasi-) norm

$$
\|x\|_{1, \infty}=\sup _{k} k x_{k}^{*} .
$$

If we consider $\alpha_{i j}=\frac{1}{j}$ and thus $\alpha_{(k-1) n+1}^{*}=\frac{1}{k}$, then the norm of a repetition $y_{k}\left(j_{1}, \ldots, j_{n}\right)=$ $j_{k}^{-1}$ is exactly

$$
\left\|\sum_{k=1}^{n} y_{k} e_{k}\right\|_{1, \infty}=\sup _{r} \frac{1}{r} \sum_{i=1}^{r} \beta_{i}
$$

Moreover, the estimate

$$
\left\|T_{t}: \ell_{1, \infty} \rightarrow \ell_{1, \infty}\right\| \leq 2 \max \{1, t\}
$$

is still valid. In this case, we see that Proposition 2.1 for $X=\ell_{1, \infty}^{n}$ is equivalent to the combinatorial estimate in Theorem 0.3.

Remark 2.4 Let $f$ be an increasing function and consider the Lorentz space

$$
\|x\|_{f, w}=\left(\sum_{k=1}^{n}\left(\frac{f(k)}{k^{\frac{1}{w}}} x_{k}^{*}\right)^{w}\right)^{\frac{1}{w}} \text { and }\|x\|_{f, \infty}=\sup _{k} f(k) x_{k}^{*} .
$$

In this context Hardy's inequality reads as follows. If

$$
\frac{f(n)}{f(k)} \leq c_{s}\left(\frac{n}{k}\right)^{\frac{1}{s}}
$$


and $q<s$, we may find $\gamma>0$ such that $p\left(\frac{1}{s}+\gamma\right)<1$. Then, for some constant $c(\gamma)$ we have

$$
\left(\sum_{k=1}^{n}\left[\frac{f(n)}{f(k)} k^{-\gamma}\right]^{q}\right)^{\frac{1}{q}} \leq c_{s} c(\gamma) n^{\frac{1}{q}-\gamma}
$$

Following [Pi, 2.1.7, $\mathrm{p}=75]$, we get

$$
\left(\sum_{k=1}^{n} x_{k}^{* q}\right)^{\frac{1}{q}} \leq c_{s} c(\gamma) \frac{n^{\frac{1}{q}}}{f(n) n^{\gamma}}\left(\sum_{k=1}^{n}\left[f(k) k^{\gamma-\frac{1}{w}} x_{k}^{*}\right]^{w}\right)^{\frac{1}{w}} .
$$

The same calculation as in $[\mathrm{Pi}, 2.1 .7]$ then yields the Hardy inequality

$$
\|x\|_{l_{f, w}} \leq\left\|\sum_{k}\left(\frac{1}{k} \sum_{j=1}^{k} x_{j}^{* q}\right)^{\frac{1}{q}} e_{k}\right\|_{l_{f, w}} \leq c^{\prime}(\gamma) c_{s}\|x\|_{l_{f, w}} .
$$

Moreover, of we assume in addition $q \leq w$, we may combine this argument with the triangle inequality in $\ell_{\frac{w}{q}}$ and deduce the $q$-convexity of $l_{f, w}$, i.e.

$$
\left\|\left(\sum_{j}\left|x_{j}\right|^{q}\right)^{\frac{1}{q}}\right\|_{l_{f, w}} \leq c_{s} c^{\prime}(\gamma)\left(\sum_{j}\left\|x_{j}\right\|_{l_{f, w}}^{q}\right)^{\frac{1}{q}} .
$$

Therefore

$$
\||x|\|=\left\||x|^{\frac{1}{q}}\right\|_{l_{f, w}}^{q}
$$

is equivalent to a norm. Given $p \geq q$, we may then apply Theorem 0.2 to $\left(\mathbb{R}^{n}, \|||||\right)$ and $\left|f_{i}\right|^{q}$ and obtain the lower estimate (with $\left(c_{s} c^{\prime}(\gamma) 1(1+\sqrt{2})\right)^{\frac{1}{q}}$ ) and the upper estimate with $\left(c_{s} c^{\prime}(\gamma) c\left(\frac{p}{q}\right)\right)^{\frac{1}{q}}$.

Now, let us consider more generally a symmetric quasi-norm satisfying the Hardy inequality

$$
\|x\|_{X} \leq\left\|\sum_{k=1}^{n}\left(\frac{1}{k} \sum_{j=1}^{k} x_{j}^{* q}\right)^{\frac{1}{q}} e_{k}\right\|_{X} \leq c(q)\|x\|_{X} .
$$

Then, we can easily modify Lemma 2.2 and deduce that for non-increasing $x$ and $y$ with

$$
\beta_{i}:=\operatorname{card}\left\{j \mid y_{j}=x_{i}\right\}
$$

we have

$$
\|y\|_{X} \leq c(q)^{\frac{1}{q}} 2^{\frac{1}{q}} \max \left\{1, \sup _{r} \frac{1}{r} \sum_{i=1}^{r} \beta_{i}\right\}^{\frac{1}{q}}\|x\|_{X} .
$$

Therefore Proposition 2.1. is still valid in this setting (using the combinatorial estimate for $\frac{p}{q}$ which is big when $q$ is small.) If $X$ is quasi-normed, there is an equivalent norm $\|||\|$ satisfying $\||x+y|\|^{r} \leq\||x|\|^{r}+\||y|\|^{r}$. Then the argument using Rosenthal's inequality easily works for $p \geq r$ and the constant $c\left(\frac{p}{r}\right)^{\frac{1}{r}}$. Therefore the upper estimate holds with $c_{0} \max \left\{\left(\frac{p}{r\left(1+\ln \frac{p}{r}\right)}\right)^{\frac{1}{r}},\left(\frac{p c_{s}}{q\left(1+\ln \frac{p}{q}\right)}\right)^{\frac{1}{q}}\right\}$ provided Hardy's inequality is available. At the time of this writing it is not clear whether the lower estimate still holds under these assumptions. 


\section{The combinatorial estimate}

The combinatorial estimate is based on a tail estimate for the variables

$$
h_{r}\left(j_{1}, . ., j_{n}\right)=\sum_{i=1}^{n} 1_{[1, r]}\left(j_{i}\right)
$$

with respect to the product probability measure on $\{1, . ., n\}^{n}$ defined by

$$
P_{\mu}\left\{\left(j_{1}, . ., j_{n}\right)\right\}=\prod_{k=1}^{n} \mu_{k j_{k}} .
$$

Note again, that

$$
\int_{\{1, \ldots, n\}^{n}}\left\|\sum_{l=1}^{n} j_{l}^{-1} e_{l}\right\|_{1, \infty}^{p} d P_{\mu}\left(j_{1}, \ldots, j_{n}\right)=\int_{\{1, \ldots, n\}^{n}} \sup _{r}\left(\frac{h_{r}}{r}\right)^{p} d P_{\mu} .
$$

Therefore, the combinatorial estimate is a special case of our main results for the weak- $\ell_{1}$ 'norm'. Here $\mu=\left(\mu_{i j}\right)_{i j}$ is assumed to be a doubly stochastic matrix and $P_{\mu}$ denotes the product probability measure on $\{1, . ., n\}^{n}$ defined by

$$
P_{\mu}\left\{\left(j_{1}, . ., j_{n}\right)\right\}=\prod_{k=1}^{n} \mu_{k j_{k}} .
$$

Let us denote by $\mathcal{C} \subset \mathbb{R}^{n}$ the set of all doubly stochastic matrices. The following lemma provides the key estimate using Birkhoff's theorem on doubly stochastic matrices. (Although the intuition for this estimate comes from the non-extremal matrix $\mu_{i j}=\frac{1}{n}$.)

Lemma 3.1 Let $\mu \in C$. Then

$$
P_{\mu}\left(h_{r}=j\right) \leq \sum_{\substack{k=0 \\
(n-j) \geq(j-k), k \leq j}}^{r}\left(\frac{k}{j}\right)^{j}\left(\frac{j-k}{n-j}\right)^{n-j}\left(\begin{array}{l}
r \\
k
\end{array}\right)\left(\begin{array}{l}
n-j \\
j-k
\end{array}\right)
$$

holds for all $1 \leq j \leq r \leq n$ and $j<n$. For $j=r=n, P_{\mu}\left(h_{n}=n\right)=1$.

Proof: The equality $h_{r}=j$ holds if and only if there is a set $B \subset\{1, . ., n\}$ of cardinality $r$ such that $j_{i} \leq r$ for $i \in B$ and $j_{i}>r$ for $i \notin B$. Using the geometric/arithmetic mean inequality, we deduce

$$
\begin{aligned}
P_{\mu}\left(h_{r}=j\right) & =\sum_{|B|=j} \prod_{i \in B}\left(\sum_{s \leq r} \mu_{i s}\right) \prod_{i \notin B}\left(\sum_{s>r} \mu_{i s}\right) \\
& \leq \sum_{|B|=j}\left(\frac{1}{j} \sum_{i \in B, s \leq r} \mu_{i s}\right)^{j}\left(\frac{1}{n-j} \sum_{i \notin B, s>r} \mu_{i s}\right)^{n-j} .
\end{aligned}
$$


Since $\mu$ is doubly stochastic, we can simplify the second term

$$
\begin{aligned}
\sum_{i \notin B, s>r} \mu_{i s} & =\sum_{i \notin B}\left(1-\sum_{s \leq r} \mu_{i s}\right)=\sum_{i=1}^{n}\left(1-\sum_{s \leq r} \mu_{i s}\right)-\sum_{i \in B}\left(1-\sum_{s \leq r} \mu_{i s}\right) \\
& =n-\sum_{s \leq r} \sum_{i=1}^{n} \mu_{i s}-j+\sum_{i \in B, s \leq r} \mu_{i s} \\
& =n-r-j+\sum_{i \in B, s \leq r} \mu_{i s} .
\end{aligned}
$$

We are lead to the function

$$
f(x)=\left(\frac{x}{j}\right)^{j}\left(1-\frac{r-x}{n-j}\right)^{n-j} .
$$

It is elementary to check that

$$
\begin{aligned}
f^{\prime \prime}(x)= & \frac{j-1}{j}\left(\frac{x}{j}\right)^{j-2}\left(1-\frac{r-x}{n-j}\right)^{n-j}+2\left(\frac{x}{j}\right)^{j-1}\left(1-\frac{r-x}{n-j}\right)^{n-j-1} \\
& +\frac{n-j-1}{n-j}\left(1-\frac{r-x}{n-j}\right)^{n-j-2}\left(\frac{x}{j}\right)^{j}
\end{aligned}
$$

which is positive on the interval $I=[\max \{0,(r+j-n)\}, \infty)$. Indeed, we note $x \geq$ $(r+j-n)$ iff $1-\frac{r-x}{n-j} \geq 0$. On the convex set $\mathcal{C}$, we consider the linear functional

$$
L(\mu)=\sum_{i \in B} \sum_{s \leq r} \mu_{i s} \geq 0
$$

Using again

$$
1-\frac{r-L(\mu)}{n-j}=\frac{1}{n-j}\left(n-r-j+\sum_{i \in B, s \leq r} \mu_{i s}\right)=\frac{1}{n-j} \sum_{i \notin B} \sum_{s>r} \mu_{i s} \geq 0,
$$

we deduce that $L(\mu) \in[\max \{0,(r+j-n)\}, \infty)$. Fixing a subset $B$, we deduce that

$$
h_{B}(\mu)=f(L(\mu))=f\left(\sum_{i \in B, s \leq r} \mu_{i s}\right)
$$

is a convex function on $\mathcal{C}$. The case $j=n$ is excluded by assumption. In particular,

$$
h(\mu)=\sum_{|B|=j} f\left(\sum_{i \in B, s \leq r} \mu_{i s}\right)
$$

is convex and attains its maximum on an extreme point. According to [Bi], the extreme points in $\mathcal{C}$ are the permutation matrices. For any permutation $\pi:\{1, . ., n\} \rightarrow\{1, . ., n\}$ we have

$$
\sum_{i \in B} \sum_{s \leq r} \delta_{\pi(i) s}=\sum_{i \in \pi^{-1}(B)} \sum_{s \leq r} \delta_{i s}=\left|\pi^{-1}(B) \cap\{1, . ., r\}\right|
$$


Since the map $B \mapsto \pi^{-1}(B)$ yields a bijection on the subsets of $\{1, . ., n\}$ of cardinality $j$, it is sufficient to consider the trivial permutation $\pi(i)=i$ and thus the identity matrix $\delta=\left(\delta_{i j}\right)$. We define $A_{r}=\{1, . ., r\}$ and get

$$
\begin{aligned}
h(\delta) & =\sum_{|B|=j}\left(\frac{1}{j} \sum_{i \in B} \sum_{s \leq r} \delta_{i s}\right)^{j}\left(\frac{1}{n-j} \sum_{i \notin B} \sum_{s>r} \delta_{i s}\right)^{n-j} \\
& =\sum_{|B|=j}\left(\frac{\left|A_{r} \cap B\right|}{j}\right)^{j}\left(\frac{\left|B^{c} \cap A_{r}^{c}\right|}{n-j}\right)^{n-j} \\
& =\sum_{k=0}^{r} \sum_{\substack{C \subset A_{r},|C|=k, D \subset A_{r}^{c},|D|=j-k}}\left(\frac{k}{j}\right)^{j}\left(\frac{j-k}{n-j}\right)^{n-j} \\
& =\sum_{k=0,(n-j) \geq(j-k), k \leq j}^{r}\left(\frac{k}{j}\right)^{j}\left(\frac{j-k}{n-j}\right)^{n-j}\left(\begin{array}{l}
r \\
k
\end{array}\right)\left(\begin{array}{l}
n-j \\
j-k
\end{array}\right) .
\end{aligned}
$$

Since $P_{\mu}\left(h_{r}=j\right)$ is majorized by $h(\delta)$, this concludes the proof.

Lemma 3.2 Let $t \geq e^{2}$ and $r<n$, then

$$
P_{\mu}\left(h_{r} \geq t r\right) \leq 2\left(\frac{e^{3}}{t}\right)^{t r}
$$

Proof: According to Lemma 3.1, we have

$$
\begin{aligned}
P_{\mu}\left(h_{r} \geq t r\right) & =\sum_{n>j \geq t r} P_{\mu}\left(h_{r}=j\right) \\
& \leq \sum_{n>j \geq t r} \sum_{k=0,(n-j) \geq(r-k)}^{\min (r, j)}\left(\frac{k}{j}\right)^{j}\left(\frac{j-k}{n-j}\right)^{n-j}\left(\begin{array}{l}
r \\
k
\end{array}\right)\left(\begin{array}{l}
n-j \\
j-k
\end{array}\right) \\
& =\sum_{k=0}^{r}\left(\begin{array}{l}
r \\
k
\end{array}\right) \sum_{n>j \geq t r, k \leq j,(n-j) \geq(r-k)}\left(\frac{k}{j}\right)^{j}\left(\frac{j-k}{n-j}\right)^{n-j}\left(\begin{array}{l}
n-j \\
j-k
\end{array}\right) .
\end{aligned}
$$

Using Stirling's formula $\left(m !=m^{m} e^{-m} \sqrt{2 \pi m} e^{\frac{\theta_{m}}{12}}, 0 \leq \theta_{m} \leq 1\right)$ we deduce (with $e^{\frac{1}{12}} \leq$ $\sqrt{2 \pi})$ that

$$
\left(\begin{array}{l}
n \\
k
\end{array}\right) \leq \frac{n^{n}}{(n-k)^{n-k} k^{k}}
$$

we get for $n-j \geq j-k$

$$
\begin{aligned}
& \left(\frac{j-k}{n-j}\right)^{n-j}\left(\begin{array}{c}
n-j \\
j-k
\end{array}\right) \\
& \quad \leq\left(\frac{j-k}{n-j}\right)^{n-j} \frac{(n-j)^{n-j}}{(n-j-(j-k))^{(n-j-(j-k))}(j-k)^{(j-k)}}
\end{aligned}
$$




$$
\begin{aligned}
& =\left(\frac{j-k}{n-j-(j-k)}\right)^{n-j-(j-k)} \\
& =\left(1+\frac{(j-k)-(n-j)+(j-k)}{n-j-(j-k)}\right)^{n-j-(j-k)} \\
& \leq \max \{1, \exp (2(j-k)-n+j)\} \leq \exp (2(j-k)) .
\end{aligned}
$$

Therefore, we have

$$
P_{\mu}\left(h_{r} \geq t r\right) \leq \sum_{k=0}^{r}\left(\begin{array}{l}
r \\
k
\end{array}\right) \exp (-2 k) \sum_{j \geq t r}\left(\frac{k e^{2}}{j}\right)^{j}
$$

For fixed $k$, we consider $f(x)=\exp (x[2+\ln k-\ln x])$ which satisfies $f^{\prime}(x)=f(x)[1+$ $\ln k-\ln x]$. On the interval $[k e, \infty)$ the function $f$ is decreasing. For $x \geq k e$, we consider

$$
g(x)=-\frac{f(x)}{\ln x-1-\ln k}
$$

and observe

$$
g^{\prime}(x)=f(x)\left(1+\frac{1}{x[\ln x-1-\ln k]^{2}}\right) \geq f(x) .
$$

Let $j_{0}$ be such that $t r \leq j_{0}<t r+1$, then we deduce from the monotonicity of $f$ and with $j_{0} \geq t r \geq e^{2} r \geq e^{2} k$ that

$$
\begin{aligned}
\sum_{j \geq j_{0}}\left(\frac{k e^{2}}{j}\right)^{j} & \leq\left(\frac{k e^{2}}{j_{0}}\right)^{j_{0}}+\int_{j_{0}}^{\infty} f(x) d x \leq\left(\frac{k e^{2}}{j_{0}}\right)^{j_{0}}+\int_{j_{0}}^{\infty} g^{\prime}(x) d x \\
& =\left(\frac{k e^{2}}{j_{0}}\right)^{j_{0}}+\frac{1}{\ln j_{0}-\ln k e}\left(\frac{k e^{2}}{j_{0}}\right)^{j_{0}} \\
& \leq\left(\frac{k e^{2}}{j_{0}}\right)^{j_{0}}+\left(\frac{k e^{2}}{j_{0}}\right)^{j_{0}}=2\left(\frac{k e^{2}}{j_{0}}\right)^{j_{0}} \leq 2\left(\frac{k e^{2}}{t r}\right)^{t r}
\end{aligned}
$$

Since $t \geq 2$, we deduce

$$
\begin{aligned}
P_{\mu}\left(h_{r} \geq t r\right) & \leq \sum_{k=0}^{r}\left(\begin{array}{l}
k \\
r
\end{array}\right) \exp (-2 k) \sum_{j \geq t r}\left(\frac{k e^{2}}{j}\right)^{j} \leq \sum_{k=0}^{r}\left(\begin{array}{l}
k \\
r
\end{array}\right) \exp (-2 k) 2\left(\frac{k e^{2}}{t r}\right)^{t r} \\
& \leq 2^{2 r}\left(\frac{e^{2}}{t}\right)^{t r} \leq\left(\frac{e^{3}}{t}\right)^{t r} .
\end{aligned}
$$

The next calculation provides the $\frac{p}{\ln p}$ term.

Lemma 3.3 Let $b \geq 1$ and $a \geq \max \left\{\frac{e^{e-1} b}{2}, 4 b^{2}\right\}$. Then

$$
e^{-2 a-1}\left(\frac{a}{1+\ln a}\right)^{a+1} \leq \int_{b}^{\infty} t^{a}\left(\frac{b}{t}\right)^{t} d t \leq(a+1)\left(\frac{2 a}{1+\ln (a)}\right)^{a}
$$


Proof: The derivative of the function $f(t)=t^{a}\left(\frac{b}{t}\right)^{t}$ is given by $f^{\prime}(t)=f(t)\left(\frac{a}{t}+\ln b-\ln t e\right)$ and thus $f$ has a unique maximum for $t_{0}$ satisfying

$$
t_{0}\left(\ln t_{0} e-\ln b\right)=a .
$$

Let us denote by $M=\sup f(t)$. Then we have

$$
M=f\left(t_{0}\right)=t_{0}^{a} e^{-t_{0}\left(\ln t_{0}-\ln b\right)}=e^{t_{0}} t_{0}^{a} e^{-t_{0}\left(\ln t_{0} e-\ln b\right)}=e^{t_{0}-a} t_{0}^{a} \leq t_{0}^{a} .
$$

Note that $g(t)=\left(\frac{b}{t}\right)^{t}$ is decreasing on $(b, \infty)$ and thus

$$
\int_{b}^{\infty} t^{a}\left(\frac{b}{t}\right)^{t} d t \geq \int_{\frac{t_{0}}{2}}^{t_{0}} t^{a}\left(\frac{b}{t}\right)^{t} d t \geq\left(\frac{t_{0}}{2}\right)^{a+1}\left(\frac{b}{t_{0}}\right)^{t_{0}}=2^{-(a+1)} t_{0} M \geq 2^{-(a+1)} e^{-a} t_{0}^{a+1} .
$$

Hence the lower estimate follows from a lower estimate of $t_{0}$. Indeed, the function $l(t)=$ $t(\ln t e-\ln b)$ is increasing on $(b, \infty)$ and it is easily checked that $b, a \geq 1 \operatorname{implies} l\left(\frac{a}{\ln e a}\right) \leq a$. Hence, $t_{0} \geq \frac{a}{\ln (e a)}$ and also $a \geq 4 b^{2}$ implies $\frac{a}{\ln e a} \geq 2 b$. We deduce

$$
\int_{b}^{\infty} t^{a}\left(\frac{b}{t}\right)^{t} d t \geq e^{-a} 2^{-(a+1)}\left(\frac{a}{1+\ln a}\right)^{a+1} .
$$

For the upper estimate of $t_{0}$ we note that $s \geq e$ implies $\frac{\ln s}{s} \leq e^{-1} \leq \frac{1}{2}$. Therefore, we deduce for $\gamma=\frac{2 e}{b}$ that

$$
l\left(\frac{2 a}{\ln (\gamma a)}\right)=\frac{2 a}{\ln (\gamma a)}[\ln (\gamma a)-\ln \ln (\gamma a)]=2 a\left[1-\frac{\ln \ln (\gamma a)}{\ln (\gamma a)}\right] \geq a .
$$

By our assumptions $\frac{a}{\ln (\gamma a)} \leq \frac{2 a}{1+\ln a}$. This yields $M \leq t_{0}^{a} \leq\left(\frac{2 a}{1+\ln (a)}\right)^{a}$. For the upper estimate of the integral, we consider $b(t)=\frac{a}{t}+\ln b-\ln (t e)$ and $h(t)=\frac{f(t)}{b(t)}$. Then the derivative of $h$ satisfies

$$
h^{\prime}(t)=f(t)\left[1-\frac{b^{\prime}(t)}{b(t)^{2}}\right]=f(t)\left[1+\frac{\frac{a}{t^{2}}+\frac{1}{t}}{b(t)^{2}}\right] \geq f(t) .
$$

Since $a(t)$ is negative for $t \geq a$, we deduce from $\frac{a}{b} \geq e$ that

$$
\begin{aligned}
\int_{b}^{\infty} t^{a}\left(\frac{b}{t}\right)^{t} d t & =\int_{b}^{a} t^{a}\left(\frac{b}{t}\right)^{t} d t+\int_{a}^{\infty} t^{a}\left(\frac{b}{t}\right)^{t} d t \\
& \leq a M+\int_{a}^{\infty} h^{\prime}(t) d t \\
& \leq a M+\frac{f(a)}{\ln (a e)-\ln b-1} \leq(a+1) M
\end{aligned}
$$

The assertion is proved. 
The next lemma is elementary. The proof uses $\frac{b}{t} \leq e^{-1}$ for $t \geq e b$ and is easier than the proof of Lemma 3.3, we omit the details.

Lemma 3.4 Let $b \geq 1$ and $a>0, d \geq b e$, then

$$
\int_{d}^{\infty}\left(\frac{b}{t}\right)^{t r} t^{a} d t \leq \frac{2}{r} \exp (-d r)\left\{\begin{array}{ll}
d^{a} & \text { if } 2 a \leq r d \\
2\left(\frac{2 a}{r}\right)^{a} & \text { if } r d \leq 2 a
\end{array} .\right.
$$

The proof of the combinatorial result is now rather a matter of calculation.

Proof of Theorem 0.3: Let $d=e^{4} \geq 2$, and choose $r_{0}$ such that $r_{0} d \leq 2(p-1) \leq$ $\left(r_{0}+1\right) d$. We also use $b=e^{3}$ and assume $p \geq 2 \max \left\{e^{e+2}, 4 e^{6}\right\}=8 e^{6}$ (which implies $\left.r_{0}+1 \geq 8 e^{2}\right)$. Then, we deduce from Lemma 3.3 and Lemma 3.4

$$
\begin{aligned}
& \int\left(\sup _{r<n} \frac{h_{r}}{r}\right)^{p} d P_{\mu} \\
& \quad=p \int_{0}^{\infty} t^{p-1} P_{\mu}\left(\bigcup_{r}\left\{h_{r}>r t\right\}\right) d t \\
& \quad \leq p \int_{0}^{d} t^{p-1} d t+\int_{d}^{\infty} t^{p-1} \sum_{r=1}^{\infty} P_{\mu}\left(h_{r} \geq r t\right) d t \\
& \leq d^{p}+\sum_{r=1}^{\infty} p \int_{d}^{\infty}\left(\frac{e^{3}}{t}\right)^{t r} t^{p-1} d t \\
& \quad \leq d^{p}+p\left(\sum_{r=1}^{r_{0}} \int_{d}^{\infty}\left(\frac{e^{3}}{t}\right)^{t r} t^{p-1} d t+\sum_{r>r_{0}}^{\infty} \int_{d}^{\infty}\left(\frac{e^{3}}{t}\right)^{t r} t^{p-1} d t\right) \\
& \quad \leq d^{p}+p\left(\sum_{r=1}^{r_{0}} p\left(\frac{2(p-1)}{1+\ln (p-1)}\right)^{p-1}+\sum_{r>r_{0}} d^{p-1} \frac{2}{r} \exp (-d r)\right) \\
& \quad \leq d^{p}+r_{0} p^{2}\left(\frac{2(p-1)}{1+\ln (p-1)}\right)^{p-1}+p d^{p-1}\left(\sum_{r \geq 1} \exp (-d r)\right) \\
& \quad \leq d^{p}+p^{3}\left(\frac{4 p}{1+\ln p}\right)^{p-1}+2 p d^{p-1} .
\end{aligned}
$$

Using the triangle inequality in $L_{p}$ and $\ell_{p}^{3}$ and $x^{\frac{1}{x}} \leq e^{\frac{1}{e}}$, we conclude the proof

$$
\begin{aligned}
\left(\int\left(\sup _{r \leq n} \frac{h_{r}}{r}\right)^{p} d P_{\mu}\right)^{\frac{1}{p}} & \leq\left(\int \frac{h_{n}{ }^{p}}{n} d P_{\mu}\right)^{\frac{1}{p}}+\left(\int\left(\sup _{r<n} \frac{h_{r}}{r}\right)^{p} d P_{\mu}\right)^{\frac{1}{p}} \\
& \leq 1+d+p^{\frac{3}{p}}\left(\frac{4 p}{1+\ln p}\right)^{\frac{p-1}{p}}+(2 p)^{\frac{1}{p}} d^{\frac{p-1}{p}} \\
& \leq 1+d+e \frac{4 p}{1+\ln p}+e d \leq 2 e^{5}+e \frac{4 p}{1+\ln p} .
\end{aligned}
$$


Remark 3.5 For $p=1$, we can use the first part of Lemma 3.4 and get the 'concrete estimate'

$$
\int \sup _{r} \frac{h_{r}}{r} d P_{\mu} \leq 2+e^{4} .
$$

Remark 3.6 As a standard application, we obtain a fairly good tail estimate. Assuming $\left\|\sum_{k=1}^{n} \alpha_{(k-1) n+1}^{*} e_{k}\right\|_{X}=1$, we have

$$
\operatorname{Prob}\left(\left\|\sum_{k=1}^{n} \alpha_{k j_{k}} e_{k}\right\|_{X}>t\right) \leq C \exp \left(-\frac{t \ln t}{C}\right)
$$

for some universal constant $C$. As usual this is obtained from Chebychev's inequality and choosing $p$ optimal. We obtain a similar behaviour for general independent functions bounded by 1 and such that $\left\|\sum_{i=1}^{n}\left(n \int_{\frac{i-1}{n}}^{\frac{i}{n}} h^{*}(s) d s\right) e_{i}\right\|_{X} \leq 1$. Of course, this behaviour is a reformulation of our main result.

Example 3.7 Let $\mu$ be the standard matrix $\mu_{i j}=\frac{1}{n}$ and $x=e_{1}$ the first unit vector and $\alpha_{i j}=\left\{\begin{array}{ll}1 & \text { if } j=1 \\ 0 & \text { else }\end{array}\right.$. Then

$$
\left(n^{n} \sum_{j_{1}, \ldots, j_{n}=1}^{n}\left\|\sum_{k=1}^{n} x\left(j_{k}\right) e_{k}\right\|_{\ell_{1}^{n}}^{p}\right)^{\frac{1}{p}}=\left(n^{n} \sum_{j_{1}, \ldots, j_{n}=1}^{n}\left\|\sum_{k=1}^{n} \alpha_{k, j_{k}} e_{k}\right\|_{\ell_{1}^{n}}^{p}\right)^{\frac{1}{p}}=\left(\int h_{1}^{p} d P_{\mu}\right)^{\frac{1}{p}}
$$

Moreover, for $p \geq p_{0}$ and $c_{0} n \geq p$

$$
\left(\int h_{1}^{p} d P_{\mu}\right)^{\frac{1}{p}} \geq c_{0} \frac{p}{1+\ln p} .
$$

In particular, the order of growth is best possible.

Proof: Since all the coefficients of $\alpha_{i j}$ are either 0 or 1 , it is clear that we count the number of events that $j_{k}=1$. This yields the first equality. For the second, we consider $2 \leq t \leq \frac{n}{2}$ and $j<t \leq j+1$ and deduce from the proof of Lemma 3.1 and Stirling's formula that

$$
\begin{aligned}
P_{\mu}\left(h_{1}>t\right) & \geq P_{\mu}\left(h_{1}=j\right)=\left(\begin{array}{c}
n \\
j
\end{array}\right) n^{-n}(n-1)^{n-j} \\
& \geq e^{-2}(4 \pi)^{-\frac{1}{2}} j^{-\frac{1}{2}} \frac{n^{n}}{j^{j}(n-j)^{n-j}} n^{-n}(n-1)^{n-j} \\
& \geq e^{-2}(4 \pi)^{-\frac{1}{2}} j^{-\left(j+\frac{1}{2}\right)} \geq e^{-2}(4 \pi)^{-\frac{1}{2}}(t-1)^{-\left(t-\frac{1}{2}\right)} .
\end{aligned}
$$


Therefore, we deduce from the proof of Lemma 3.3 that for $p \geq p_{0}$ and $\frac{n}{2}-1 \geq \frac{4\left(p-\frac{3}{2}\right)}{1+\ln \left(p-\frac{3}{2}\right)}$, we have

$$
\begin{aligned}
\int h_{1}^{p} d P_{\mu} & =p \int_{0}^{\infty} t^{p-1} P_{\mu}\left(h_{1}>t\right) d t \geq \frac{p}{e^{2}(4 \pi)^{\frac{1}{2}}} \int_{2}^{\frac{n}{2}} t^{p-1}(t-1)^{-\left(t-\frac{1}{2}\right)} d t \\
& =\frac{p}{e^{2}(4 \pi)^{\frac{1}{2}}} \int_{1}^{\frac{n}{2}-1}(t+1)^{p-1} t^{-\left(t+\frac{1}{2}\right)} d t \geq \frac{p}{e^{2}(4 \pi)^{\frac{1}{2}}} \int_{1}^{\frac{n}{2}-1} t^{p-\frac{3}{2}} t^{-t} d t \\
& \geq \frac{p}{e^{2}(4 \pi)^{\frac{1}{2}}} e^{-2 p-2}\left(\frac{p-\frac{3}{2}}{1+\ln \left(p-\frac{3}{2}\right)}\right)^{p-\frac{1}{2}} .
\end{aligned}
$$

This yields the assertion.

Remark 3.8 By complex interpolation, we see that for $1 \leq q \leq \infty, 1 \leq p \leq \infty$, we have

$$
\left(n^{-n}\left\|\sum_{j_{1}, \ldots, j_{n}=1}^{n} \sum_{k=1}^{n} \alpha_{k j_{k}} e_{k}\right\|_{q}^{p}\right)^{\frac{1}{p}} \leq c_{p}^{\frac{1}{q}}\left\|\sum_{k=1}^{n} \alpha_{(k-1) n+1}^{*} e_{k}\right\|_{q} .
$$

Again the same example shows that for $c_{0} p \geq q$ this behaviour is best possible.

\section{Application to noncommutative $L_{p}$-spaces}

This part is devoted to application in terms of non-commutative version of symmetric spaces. Indeed, if $X$ is a symmetric sequence space with basis $\left(e_{k}\right)$, one may define

$$
S_{X}=\left\{a \in B\left(\ell_{2}\right) \mid\left\|\sum_{k} s_{k}(a) e_{k}\right\|_{X}<\infty\right\} .
$$

Here $\left(s_{k}(a)\right)$ denotes the sequence of singular values, i.e. the non-increasing rearrangement of the sequence of eigenvalues of $\lambda_{k}\left(\left(a^{*} a\right)^{\frac{1}{2}}\right)$. Then the norm of $a \in S_{X}$ is given by

$$
\|x\|_{S_{X}}=\left\|\sum_{k} s_{k}(a) e_{k}\right\|_{X}
$$

We refer to [Wo, Proposition III.G.11] and [LT, Proposition 2.a.5] to the non-trivial fact that this provides indeed a norm, see $[\mathrm{S}, \mathrm{DDP}]$ for more information. We use the notation $S_{X}^{n}$ for the subspace of $n \times n$ matrices in $S_{X}$. $M_{n}$ denotes the space of $n \times n$ matrices with the operator norm. Let us also recall the more general definition of noncommutative $L_{p}$ spaces. If $N$ is a von Neumann algebra and $\tau$ is a normal, faithful, semifinite trace, then the $L_{p}$-norm of a $\tau$-measurable operator $x$ is defined as

$$
\|x\|_{L_{p}(N, \tau)}=\left[\tau\left(\left(x^{*} x\right)^{\frac{p}{2}}\right)\right]^{\frac{1}{p}} .
$$


We refer to $[\mathrm{Ne}]$ for basic properties and to $[\mathrm{FK}]$ for more information. The definition of $L_{p}$ spaces was extended to non-semifinite von Neumann algebras by Connes [Co] and Haagerup [Ha]. We only need the very basic properties b),c) (see [Te, Ps, JR]) and the recent result of Raynaud that the class of non-commutative $L_{p}$ spaces is closed by ultra-products (noted as a) below).

a) The class of non-commutative $L_{p}$ spaces is closed by ultra-products.

b) $L_{p}(N)$ decomposes into $L_{p}(N)_{s a}+i L_{p}(N)_{s a}$ such that

$$
\|a+i b\|_{p} \sim_{2} \max \left\{\|a\|_{p},\|b\|_{p}\right\} .
$$

c) For all $m \in \mathbb{N}$, there is a distinctive norm \|\|$_{p}$ on $L_{p}\left(M_{m} \otimes N\right)$ such that for every unitary $u \in M_{m} \bar{\otimes} N$ and $x \in L_{p}\left(M_{m} \otimes N\right)$

$$
\left\|u^{*} x u\right\|_{p}=\|x\|_{p} .
$$

Moreover, for a diagonal n element $x=\left(x_{i j}\right) \in L_{p}\left(M_{m} \otimes N\right)$ (i.e. $x_{i j}=0$ for $i \neq j$ ), we have

$$
\|x\|_{\left.L_{p}\left(M_{m} \otimes N\right)\right)}=\left(\sum_{i=1}^{n}\left\|x_{i i}\right\|_{L_{p}(N)}^{p}\right)^{\frac{1}{p}} .
$$

Lemma 4.1 Let $1 \leq p<\infty, N$ be a von Neumann algebra, $X$ be a symmetric sequence space and $\iota: X \rightarrow L_{p}(N)$ be an embedding. Then there is an embedding of $S_{X}^{n}$ into $L_{p}\left(M_{n^{n}} \otimes(N \oplus N)\right)$.

Proof: Let $v: X \rightarrow L_{p}(N)$ be an isomorphism into its image, then we can decompose $v=\left(v_{1}, v_{2}\right)$ into two real-linear maps $v_{1}(x)=\frac{v(x)+v(x)^{*}}{2}$ and $v_{2}(x)=\frac{v(x)-v(x)^{*}}{2 i}$ such that $\|v(x)\|_{p} \sim_{2}\left(\left\|v_{1}(x)\right\|_{p}^{p}+\left\|v_{2}(x)\right\|_{p}^{p}\right)^{\frac{1}{p}}$. Note that the new map $\left(v_{1}, v_{2}\right): X \rightarrow L_{p}(N \oplus N)$ maps $X$ into the selfadjoint part and is still a real-linear isomorphism. Thus, we may assume that $v(X) \subset L_{p}(N)_{s a}$. Let $a \in M_{n}$ be a selfadjoint matrix. Let $D_{\sigma}$ be the diagonal matrix given by the sequence $\sigma=\left(s_{k}(a)\right)_{k=1}^{n}$ and $u$ be unitary such that $a=u^{*} D_{\sigma} u$. We deduce from Proposition 2.1 applied to the matrix $\alpha_{i j}=\sigma_{j}(a)$.

$$
\begin{aligned}
\|a\|_{X} & =\left\|\sum_{k=1}^{n} s_{k}(a) e_{k}\right\|_{X} \sim_{c_{p}}\left(n^{-n} \sum_{j_{1}, \ldots, j_{n}=1}^{n}\left\|\sum_{k=1}^{n} s_{j_{k}} e_{k}\right\|_{X}^{p}\right)^{\frac{1}{p}} \\
& \sim_{c(v)} n^{-\frac{n}{p}}\left(\sum_{j_{1}, \ldots, j_{n}=1}^{n}\left\|\sum_{k=1}^{n} s_{j_{k}} v\left(e_{k}\right)\right\|_{L_{p}(N)}^{p}\right)^{\frac{1}{p}} \cdot
\end{aligned}
$$

Here $c(v)=\|v\|\left\|v^{-1}: \operatorname{Im}(v) \rightarrow X\right\|$ only depends on $v$. For fixed $k$, we consider the map $\pi_{k}: M_{n} \otimes M_{n^{n}}$ given by

$$
\pi_{k}(x)=1 \otimes \cdots \otimes \underbrace{x}_{k \text {-th position }} 1 \otimes \cdots \otimes 1
$$


Note that $\pi_{k}\left(D_{\sigma}\right)$ is a diagonal matrix $D_{\sigma_{k}}$ in $M_{n^{n}}$ with entry $\sigma_{k}\left(j_{1}, \ldots, j_{n}\right)=\sigma_{j_{k}}$. Therefore, we have shown that for all diagonal matrices $x=D_{\sigma}$ we have

$$
\left\|D_{\sigma}\right\|_{X} \quad \sim_{c_{p} c(v)} n^{-\frac{n}{p}}\left\|\sum_{k=1}^{n} \pi_{k}\left(D_{\sigma_{k}}\right) \otimes v\left(e_{k}\right)\right\|_{L_{p}\left(M_{n^{n}} \otimes N\right)} .
$$

However, $a=u^{*} D_{\sigma} u$ and

$$
\left(u^{*} \otimes \cdots \otimes u^{*}\right) \pi_{k}\left(D_{\sigma}\right)(u \otimes \cdots \otimes u)=\pi_{k}(a)
$$

Since $(u \otimes \cdots \otimes u) \otimes 1$ is a unitary in $M_{n^{n}} \otimes N$, we deduce

$$
\|a\|_{X} \sim_{c_{p} c(v)} n^{-\frac{n}{p}}\left\|\sum_{k=1}^{n} \pi_{k}(a) \otimes v\left(e_{k}\right)\right\|_{L_{p}\left(M_{n^{n}} \otimes N\right)} .
$$

for all selfadjoint matrices $a \in M_{n}$. Note that $\left(\right.$ since $\left.v(X) \subset L_{p}(N)_{s a}\right)$ the map $T: S_{X}^{n} \rightarrow$ $L_{p}\left(M_{n^{n}} \otimes N\right)$ defined by

$$
T(a)=n^{-\frac{n}{p}} \sum_{k=1}^{n} \pi_{k}(a) \otimes v\left(e_{k}\right)
$$

maps selfadjoint elements to selfadjoint elements. Thus for arbitrary $x=a+i b$ we have

$$
T(x)=T(a)+i T(b)
$$

By $b$ ) and the fact that

$$
\|x\|_{S_{X}} \sim_{2} \max \left\{\|a\|_{S_{X}},\|b\|_{S_{X}}\right\}
$$

we see that $T$ still defines an isomorphism with constant $16 c_{p}\|v\|\left\|v^{-1}: \operatorname{Im}(v) \rightarrow X\right\|$.

Remark 4.2 The same remark shows that $S_{q}^{n}=S_{\ell_{q}}^{n}$ embeds into the vector-valued noncommutative $L_{p}$-space $S_{p}^{n^{n}}\left[\ell_{q}^{n}\right]$ defined by Pisier. Indeed,

$$
\|a\|_{S_{q}^{n}} \sim_{c_{p}} n^{-\frac{n}{p}}\left\|\sum_{k=1}^{n} \pi_{k}(a) \otimes e_{k}\right\|_{S_{p}^{n^{n}}\left[\ell_{q}^{n}\right]} .
$$

We refer to [Ps] for a definition of the norm in this space which is $\ell_{p}^{n^{n}}\left(\ell_{q}^{n}\right)$ on the diagonal and satisfies

$$
\left\|\sum_{k=1}^{n} u x_{k} v \otimes e_{k}\right\|_{S_{p}^{n^{n}}\left[\ell_{q}^{n}\right]}=\left\|\sum_{k=1}^{n} x_{k} \otimes e_{k}\right\|_{S_{p}^{n^{n}}\left[\ell_{q}^{n}\right]}
$$

for all unitaries $u, v \in M_{n^{n}}$. The embedding obtained in this way is not a complete isomorphism for $q=1$. 
Proof: Since $\|a\|_{1} \sim \max \left\{\left\|\frac{a+a^{*}}{2}\right\|_{q},\left\|\frac{a-a^{*}}{2 i}\right\|_{q}\right\}$, it suffices to prove the equivalence of norms for selfadjoint matrices. Using the unitary invariance, it suffices (as above) to prove it for diagonal matrices. In that case it is a special case of Proposition 2.1. Now, let us indicate why this is not a cb-isomorphism for $q=1$. We will freely use results from [Ps]. Let us use the notation $\tau_{m}=\frac{1}{m} t r$ for the normalized trace. We consider the element

$$
x=\sum_{i, j=1}^{n} e_{i j} \otimes e_{i j} \in L_{p}\left(M_{n}, \tau_{n} ; S_{1}^{n}\right) .
$$

Using simple facts about the Haagerup tensor product, we have

$$
\|x\|_{L_{p}\left(M_{n}, \tau_{n} ; S_{1}^{n}\right)}=n^{-\frac{1}{p}}\|i d\|_{S_{p}^{n}\left[S_{1}^{n}\right]}^{2}=1 .
$$

Due to the decomposition

$$
x=\left(\sum_{i=1}^{n} e_{(i, i), 1}\right)\left(\sum_{j=1}^{n} e_{1,(j, j)}\right)
$$

we see that $x$ is positive. Here $e_{(i j),(k, l)}$ corresponds to the matrix units in $M_{n^{2}}$. Positivity (see e.g. [Ju]) and the Burkholder/Rosenthal inequality (see [JX]) imply

$$
\begin{aligned}
& \left\|\sum_{k=1}^{n} i d \otimes \pi_{k}(x) \otimes e_{k}\right\|_{L_{p}\left(M_{n^{n+1}}, \tau_{n^{n+1} ;} ; \ell_{1}^{n}\right)}^{\frac{1}{2}}=\left\|\sum_{k=1}^{n} i d \otimes \pi_{k}(x)\right\|_{L_{p}\left(M_{n^{n+1}}, \tau_{n^{n+1}}\right)}^{\frac{1}{2}} \\
& =\left\|\sum_{k=1}^{n}\left(i d \otimes \pi_{k}\left(x^{\frac{1}{2}}\right)\right) \otimes e_{k, 1}\right\|_{L_{2 p}\left(M_{n^{n+1}}, \tau_{n^{n+1}} ; \ell_{2}^{c}\right)} \\
& \sim_{c_{2 p}} \max \left\{n^{\frac{1}{2 p}}\left\|x^{\frac{1}{2}}\right\|_{L_{2 p}\left(M_{n^{2}}, \tau_{n}\right)}, \sqrt{n}\left\|\sum_{i, j=1}^{n} e_{i j} \tau_{n}\left(e_{i j}\right)\right\|_{L_{p}\left(M_{n}, \tau_{n}\right)}^{\frac{1}{2}}\right\} \\
& =\max \left\{n^{\frac{1}{2 p}}\|x\|_{L_{p}\left(M_{n^{2}}, \tau_{n^{2}}\right)}^{\frac{1}{2}},\left\|\sum_{i=1}^{n} e_{i i}\right\|_{L_{p}\left(M_{n}, \tau_{n}\right)}^{\frac{1}{2}}\right\} \\
& =\max \left\{\|x\|_{L_{p}\left(M_{n}, \tau_{n} ; S_{p}^{n}\right)}^{\frac{1}{2}}, 1\right\} .
\end{aligned}
$$

Now, we use the fact that the inclusion $i d: S_{1}^{n} \rightarrow S_{p}^{n}$ is not completely bounded and this is witnessed by the element $x$. Indeed, according to (5), we see that $x$ represents a rank 1 matrix and thus get

$$
\|x\|_{L_{p}\left(M_{n}, \tau_{n} ; S_{p}^{n}\right)}=n^{-\frac{1}{p}}\|x\|_{S_{p}^{n^{2}}}=n^{-\frac{1}{p}}\left\|\sum_{i=1}^{n} e_{(i, i), 1}\right\|_{2 p}^{2}=n^{1-\frac{1}{p}} .
$$

Therefore, the cb-norm of the map $T: S_{1}^{n} \rightarrow L_{p}\left(M_{n^{n}}, \tau_{n^{n}} ; \ell_{1}^{n}\right)$ satisfies

$$
n^{1-\frac{1}{p}} \leq c_{p}\left\|i d_{L_{p}\left(M_{n}, \tau_{n}\right)} \otimes T: L_{p}\left(M_{n}, \tau_{n} ; S_{1}^{n}\right) \rightarrow L_{p}\left(M_{n^{n+1}}, \tau_{n^{n+1}} ; \ell_{1}^{n}\right)\right\| \leq c_{p}\|T\|_{c b} .
$$

(Actually it is not very difficult to show that the upper estimate holds too.) The assertion is proved. 
For the proof of Theorem 0.4, we need some facts about symmetric spaces with finite cotype.

Lemma 4.3 Let $X$ be a symmetric sequence space such that $X$ admits an embedding into $L_{p}(N)$ for some $1 \leq p<\infty$, then for all $x \in X$

$$
\|x\|_{X}=\sup _{n}\left\|\sum_{k=1}^{n} x_{k} e_{k}\right\|_{X} .
$$

Moreover, the sequences with finite support are dense.

Proof: Since $L_{p}(N)$ has cotype $\max (2, p)<\infty$ (see [TJ, Fa]), we see that $X$ cannot contain a copy of $c_{o}$ on disjoint blocks. Therefore $X$ is $\sigma$-order complete (cf [LT, Proposition 1.a.5]). From [LT, Proposition 1.a.7], we deduce that $X$ is $\sigma$-order continuous. Let $x \geq 0$ be an element in $X$ and consider $y_{n}=x-\sum_{k=1}^{n} x_{k} e_{k}$. Then $y_{n}$ converges to 0 everywhere and thus

$$
\lim _{n}\left\|y_{n}\right\|_{X}=0
$$

This implies both assertions.

Lemma 4.4 Let $X \subset c_{o}$ be a symmetric sequence space satisfying the Fatou property (6). For $k \in \mathbb{N}$, we denote by $p_{k}$ the projection onto the first $k$ unit vectors in $\ell_{2}$. Then

$$
\|a\|_{S_{X}}=\sup _{k}\left\|p_{k} a p_{k}\right\|_{X}
$$

Proof: Since $s_{j}\left(p_{k} a p_{k}\right) \leq s_{j}(a)$ we only have to show" $\leq "$. Since $X \subset c_{o}$, we may assume that $a$ is compact (and using the spectral theorem for the compact operator $\left(a^{*} a\right)^{\frac{1}{2}}$ ) and thus, we may write $a=u D_{\sigma} v$ for unitaries $u, v$ and a diagonal operator $D_{\sigma}$. In particular, we may find projections $e_{n}=u p_{n} u^{*}$ and $f_{n}=v^{*} p_{n} v$ of rank $n$ such that

$$
\left\|u p_{n} D_{\sigma} p_{n} v\right\|_{S_{X}}=\left\|e_{n} a f_{n}\right\|_{S_{X}}
$$

Since $f_{n}$ and $e_{n}$ have finite ranks, we see that

$$
\lim _{k}\left\|\left(1-p_{k}\right) f_{n}\right\|=0=\lim _{k}\left\|e_{n}\left(1-p_{k}\right)\right\|
$$

By the triangle inequality, we deduce

$$
\begin{aligned}
& \left\|e_{n} a f_{n}\right\|_{S_{X}} \\
& \quad=\lim _{k}\left\|e_{n} p_{k} a p_{k} f_{n}+e_{n}\left(1-p_{k}\right) a p_{k} f_{n}+e_{n} p_{k} a\left(1-p_{k}\right) f_{n}+e_{n}\left(1-p_{k}\right) a\left(1-p_{k}\right) f_{n}\right\| \\
& \quad \leq \limsup _{k}\left\|e_{n} p_{k} a p_{k} f_{n}\right\|_{S_{X}}+\limsup _{k} 2\left\|e_{n}\left(1-p_{k}\right)\right\|_{\infty}\|a\|_{S_{X}}+\|a\|_{S_{X}}\left\|\left(1-p_{k}\right) f_{n}\right\|_{\infty} \\
& \quad \leq \limsup _{k}\left\|p_{k} a p_{k}\right\|_{S_{X}} .
\end{aligned}
$$


Since $X$ is supposed to satisfy (6), we have

$$
\|a\|=\sup _{n}\left\|p_{n} D_{\sigma} p_{n}\right\|_{X} \leq \sup _{n}\left\|e_{n} a f_{n}\right\|_{S_{X}} \leq \sup _{k}\left\|p_{k} a p_{k}\right\|_{S_{X}} .
$$

The assertion is proved.

Proof of Theorem 0.4: Since the space of diagonal matrices in $S_{X}$ is $X$, it suffices to show that $S_{X}$ embeds into some $L_{p}(N)$ if there is an isomorphism $v: X \rightarrow L_{p}(N)$. Since $L_{p}(N)$ has cotype $\max (2, p)$, we have in particular, that $X \subset c_{o}$. According to Lemma 4.3 and Lemma 4.1, we see that

$$
\|a\|_{S_{X}}=\sup _{n}\left\|p_{n} a p_{n}\right\|_{S_{X}}=\limsup _{n}\left\|p_{n} a p_{n}\right\|_{S_{X}^{n}} .
$$

Let $\mathcal{U}$ be an ultrafilter on the integers. According to Lemma 4.4, the mapping

$$
\iota: S_{X} \rightarrow \prod_{n, \mathcal{U}} S_{X}^{n} \quad, \quad \iota(x)=\left(\left(x_{i j}\right)_{i, j=1}^{n}\right)
$$

is an isometric isomorphism. Due to Lemma 4.1, we may find $N_{n}$ and $T_{n}: S_{X}^{n} \rightarrow L_{p}\left(N_{n}\right)$ such that $\left\|T_{n}\right\| \leq 1$ and $\left\|T_{n}: \operatorname{Im}\left(T_{n}\right) \rightarrow S_{X}^{n}\right\| \leq 16 c_{p} c(v)$. Hence,

$$
T: S_{X} \rightarrow \prod_{n, \mathcal{U}} L_{p}\left(N_{n}\right)
$$

is an isomorphism and the assertion is proved using Raynaud's [Ra] result (stated as a) above).

\section{References}

[Bi] G. Birkhoff: Three observations on linear algebra; Univ. Nac. Tucumán. Revista A. 5, (1946). 147-151.

[Bu] D.L. Burkholder: Martingale theory and harmonic analysis in Euclidean spaces, Harmonic analysis in Euclidean spaces (Proc. Sympos. Pure Math., Williams Coll., Williamstown, Mass., 1978), Part 2, pp. 283-301, Proc. Sympos. Pure Math., XXXV, Part, Amer. Math. Soc. Providence, R.I., 1979.

[Co] A. Connes: Sur la théorie non-commutative de l'integration; Lecture Notes in Math. 725, Springer, Berlin, 1979.

[DDP] P. Dodds, T. Dodds And B. De PAGTER; Noncommutative Banach function spaces; Math. Z. 201 (1989), no. 4, 583-597.

[Fa] T. FACK: Type and cotype inequalities for noncommutative $L^{p}$-spaces; J. Operator Theory 17 (1987), no. 2, 255-279.

[FK] T. FACK AND H. KOSAKI: Generalized s-numbers of $\tau$-measurable operators; Pacific J. Math. 123 (1986), no. 2, 269-300. 
[GE] S. GeISS: $\mathrm{BMO}_{\psi}$-spaces and applications to extrapolation theory; Studia Math. 122 (1997), no. 3, 235-274.

[GLSW] Y. Gordon, A. Litvak, C. Schütt and E. Werner: Orlicz Norms of Seqeunces of Random Variables; to appear in Annals of Probability.

[Ha] U. HAagerup: $L^{p}$-spaces associated with an arbitrary von Neumann algerba; Algeèbres d'operateurs et leurs applicatons en physique methématique (CNRS, No. 274, Marseille 20-24 juin 1977), 175-185, Paris 1979.

[Hi] P. Hitczenko: On a domination of sums of random variables by sums of conditionally independent ones; Ann. Probab. 22 (1994), no. 1, 453-468.

[HMS] P. Hitczenko And S. Montgomery-Smith: Measuring the magnitude of sums of independent random variables; Ann. Probab. 29 (2001), no. 1, 447-466.

[JS] W.B. Johnson and G. Schechtman: Sums of independent random variables in rearrangement invariant function spaces; Ann. Probab. 17 (1989), no. 2, 789-808.

[Ju] M. Junge: Doob's inequality for non-commutative martingales; J. reine angew. Math. 549 (2002), 149-190.

[JR] M. Junge, M. And Z.J. Ruan: Approximation Properties for Noncommutative $L_{p}$-Spaces Associated with Discrete Groups, to appear in Duke Math. Journal.

[JX] M. JUnge AND Q. XU: Burkholder/Rosenthal inequalities for noncommutative martingales; accepted for publication in Ann. of Probab.

[JSZ] W. B. Johnson, G. Schechtman and J. Zinn: Best constants in moment inequalities for linear combinations of independent and exchangeable random variables; Ann. Probab. 13 (1985), no. 1, 234-253.

[KSI] S. KWAPIEŃ AND C. Schü: Some combinatorial and probabilistic inequalities and their application to Banach space theory; Israel seminar on geometrical aspects of functional analysis (1983/84), XI-2, 21 pp., Tel Aviv Univ., Tel Aviv, 1984.

[KSII] S. KWAPIEŃ AND C. Schü: Some combinatorial and probabilistic inequalities and their application to Banach space theory. II; Studia Math. 95 (1989), no. 2, 141-154.

[KSz] S. Kwapień AND J. Szulga: Hypercontraction methods in moment inequalities for series of independent random variables in normed spaces Ann. Probab. 19 (1991), no. 1, 369-379.

[LT] J. Lindenstrauss And L. Tzafriri: Classical Banach spaces. II. Function spaces; Ergebnisse der Mathematik und ihrer Grenzgebiete [Results in Mathematics and Related Areas], 97. Springer-Verlag, Berlin-New York, 1979.

[MSe] S. Montgomery-Smith and E. Semenov: Random rearrangements and operators; Voronezh Winter Mathematical Schools, 157-183, Amer. Math. Soc. Transl. Ser. 2, 184, Amer. Math. Soc., Providence, RI, 1998.

[MS] S. Montgomery-Smith: Rearrangement invariant norms of symmetric seqeunces nomrs of independent seqeunces of random variables; preprint 2002. 
[Ne] E. Nelson: Notes on non-commutative integration; J. Funct. Analayis 15 (1974), 103-116.

[Ro] H. Rosenthal: On the subspaces of $L^{p}(p>2)$ spanned by sequences of independent random variables; Israel J. Math. 8 (1970) 273-303.

[Pi] A. Pietsch: Eigenvalues and s-numbers; Cambridge Studies in Advanced Mathematics, 13. Cambridge University Press, Cambridge, 1987.

[Ps] G. PISIER; Non-commutative vector valued $L_{p}$-spaces and completely $p$ summing maps; Astrisque No. 247 (1998)

[Ra] Y. RAYNAUD: On ultrapowers of non-commutative $L_{p}$ spaces; to appear in J. of Operator theory.

[S] F. A. Sukochev: Construction of noncommutative symmetric spaces; (Russian) Dokl. Akad. Nauk UzSSR 1986, no. 8, 4-6.

[Te] M. TerP: $L^{p}$ spaces associated with von Neumann algebras Notes, Math. Institute, Copenhagen Univ. 1981.

[TJ] N. TomczaK-Jaegermann: Uniform convexity of unitary ideals; Israel J. Math. 48 (1984), no. 2-3, 249-254.

[Wo] P. Wojtaszczyk: Banach spaces for analysts; Cambridge Studies in Advanced Mathematics, 25. Cambridge University Press, Cambridge, 1991. 